\title{
Inhibition of bacterial binding through dysfunction of bacterial adhesion pili
}

\author{
Matthew Doran ${ }^{1}$ and Esther Bullitt ${ }^{2}$
}

${ }^{1}$ Boston University School of Medicine, Allston, Massachusetts, United States, ${ }^{2}$ Boston University School of Medicine, United States

Enterotoxigenic Escherichia coli (ETEC) cause substantial morbidity by producing diarrhea in developing countries, as well as being the leading cause of traveler's diarrhea ${ }^{1,2}$. Importantly, in infants, infection by ETEC can cause a host of problems such as chronic malnutrition, stunted growth, and impaired cognitive development ${ }^{3}$. Although cases of diarrheal disease have waned recently, the persistently high incidence results in hundreds of thousands of deaths each year ${ }^{1}$. Developing treatments to stop ETEC is of paramount importance.

ETEC are extracellular pathogens that colonize the small intestine to initiate pathogenesis by adhering to the epithelial wall, a process mediated by adhesion pili ${ }^{4}$. CFA/I, the most commonly occurring pilus in ETEC, is made up of helically arranged subunits whose elongated assemblies extend from the bacterial surface and terminate at a specialized adhesin tip that makes contact with epithelial cells ${ }^{5}$.

Adhesion pili's main role is to keep the bacteria attached to the cell surface under the strong fluid forces in the intestines. The structure of these filaments is essential in maintaining this adhesion. The CFA/I pilus is assembled from subunits as a single-start helix that consists of two important interactions. The first is a strong interaction between adjacent subunits in which the $\mathrm{N}$-terminus of one subunit is donated to the next in order to fill a groove in the $\beta$-strand arrangement of the pilin quaternary structure ${ }^{6}$. The second is between subunits above and below a single monomer. This weak layer-to-layer interaction breaks under force, causing the pili to unwind. Pili unwinding decreases the shear force on the adhesin tip and promotes sustained attachment to the epithelium. Once the forces decrease, the interaction is restored, and the pili re-coil. This transition is builtin to the structure of adhesion pili, and is an essential process in the pathogenesis of ETEC ${ }^{7}$.

Humans have developed innate protections that serve therapeutic roles to neutralize bacteria before they initiate disease. One such protection is a class of antimicrobial, histidine-rich peptides found in saliva, called histatins. We have shown that histatin-5 directly binds to CFA/I pili and markedly reduces the amount of adhered ETEC to cultured monolayers (Figure 1$)^{8}$. In addition, the peptide was shown to increase the stiffness of the pili, likely disrupting its winding/unwinding ability that allows it to buffer the shear forces experienced in the intestines, causing the bacterium to wash away to prevent colonization.

Inhibition of ETEC adhesion by histatin-5 is known, but the molecular mechanism for this inhibition is not understood. This information is essential for 1) understanding how histatin-5 binding confers ultrastructural changes in the CFA/I pili and 2) determining histatin's potential use as a therapeutic. To elucidate the molecular mechanism behind histatin-5's anti-ETEC action, we aim to solve the structure of the CFA/I pili in complex with histatin-5 using cryoelectron microscopy (cryo-EM).

Cryo-EM images comparing CFA/I with bound histatin-5 to CFA/I alone show clear ultrastructure differences. The addition of histatin-5 produces shorter and straighter filaments, consistent with the observation that CFA/I pili treated with histatin- 5 become stiffer. In addition, reference-free $2 \mathrm{D}$ class averages ${ }^{9}$ show that in the absence of histatin-5, CFA/I pili have a visible central channel that winds around the helical axis (Figure 2A). However, the addition of histatin-5 results in CFA/I pili with visible density at filament core (Figure $2 \mathrm{~B}$ ). These data provide evidence that histatin-5's action occurs through interactions with subunits in the interior of the filament assembly. We are in the process of collecting and processing high-resolution data to visualize the 
near-atomic structure of the histatin-5 binding site. In the meantime, we have utilized protein-protein docking methods to provide insights into the pili-peptide interaction.

Our protein-protein docking using ClusPro investigates potential binding sites for the histatin-5 peptide to the CFA/I pili (Figure 2C). This program uses shape complementarity, residue-to-residue electrostatic interactions, and van der Waals chemical potentials to rank likely binding sites between two proteins ${ }^{10}$. The histatin-5 peptide, modeled from NMR data ${ }^{11}$, was docked to a six subunit pilus, constructed from our most recent cryoEM reconstruction of the CFA/I filament ${ }^{6}$. Consistent with the $2 \mathrm{D}$ class averages, the best ranked pose places the peptide in the hollow core of the filament, displaying many contacts between the basic peptide and acidic interior of CFA/I. The most abundant interactions between the peptide and the pilin subunits occur between subunits along the helical axis, strengthening the weak, layer-to-layer interaction. By doing so, the unwinding action is expected to be compromised, presumably reducing the bacterium's ability to withstand the forces acting on the adhesin tip. This mechanism of action is being tested through our cryo-EM structure determination of the CFA/I pili in complex with the histatin-5 peptide.

Combining ex vivo results, cryo-EM data, and protein-protein docking methods, we have shown that the salivary peptide, histatin-5, inhibits adhesion of ETEC to intestinal epithelium cells, and stiffens the adhesion pili CFA/I. We propose a molecular mechanism where the peptide binds to the inner core of the pilus, strengthening vertical associations between subunits to disrupt the vital winding/unwinding mechanism. In further work, we will solve the near-atomic cryo-EM structure of CFA/I with bound histatin-5, thereby determining the histatin-5 binding site and the molecular mechanism by which histatin-5 inhibits ETEC infection. Armed with this information, therapeutic peptides could be designed to increase binding affinity, improving protection against ETEC-induced diarrheal disease.
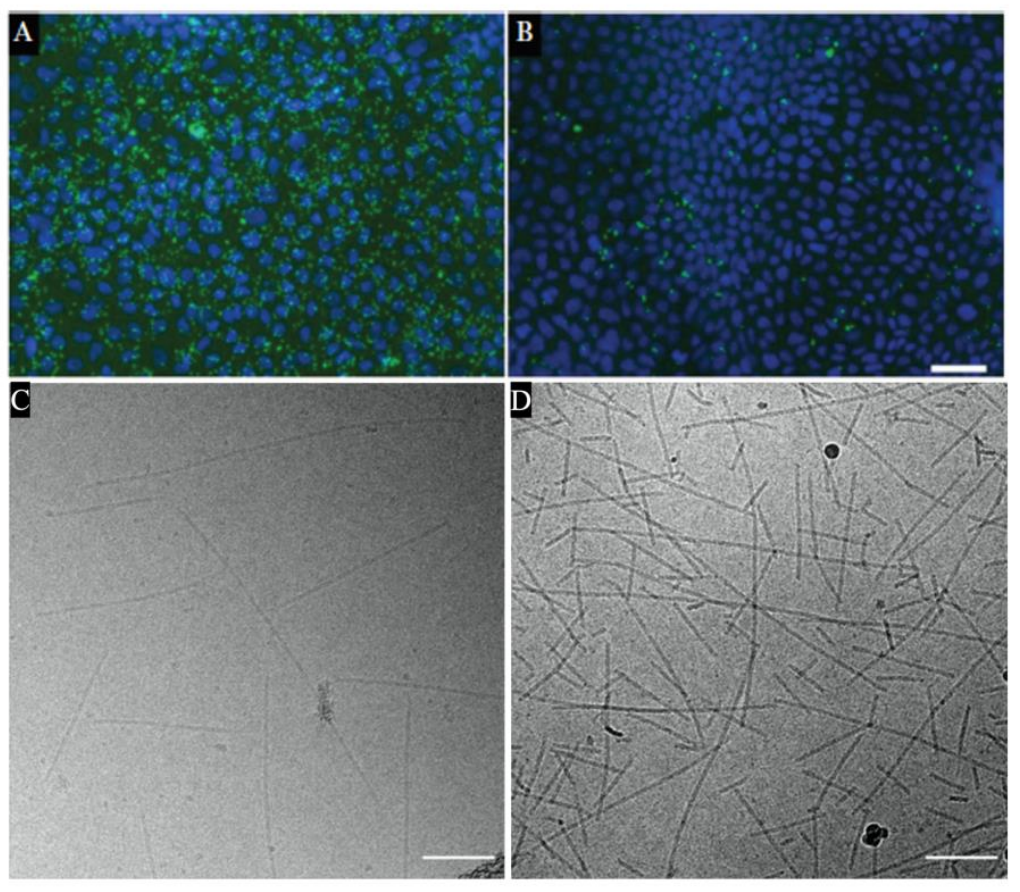

Figure 1. Histatin-5 inhibits binding of diarrhea-causing ETEC and alters the ultrastructure of CFA/I pili expressed on the ETEC bacterial surface. A, B). Histatin-5 decreases the adherence of ETEC to Caco-2 tissue culture cells. Fluorescence microscopy of Caco-2 cells inoculated with ETEC in the absence (A) and presence 
(B) of $100 \mu \mathrm{M}$ histatin-5 show reduced bacterial binding in the presence of the salivary peptide. Caco-2 nuclei were stained with 4',6-diamidino-2-phenylindole (blue) and ETEC were stained with AlexaFluor 488 labelled anti-ETEC antibody (green); from Reference 8. C, D). Histatin-5 disrupts the ultrastructure of the CFA/I filaments. Images of frozen-hydrated CFA/I pili were collected on a Tecnai TF20 at a defocus of $\sim 5$ $\mu \mathrm{m}$ without histatin-5 (C) and with histatin-5 (D). In the presence of histatin-5 the pilus filaments are shorter and appear straighter, as compared to control filaments. Scalebar, $50 \mathrm{~nm}$.
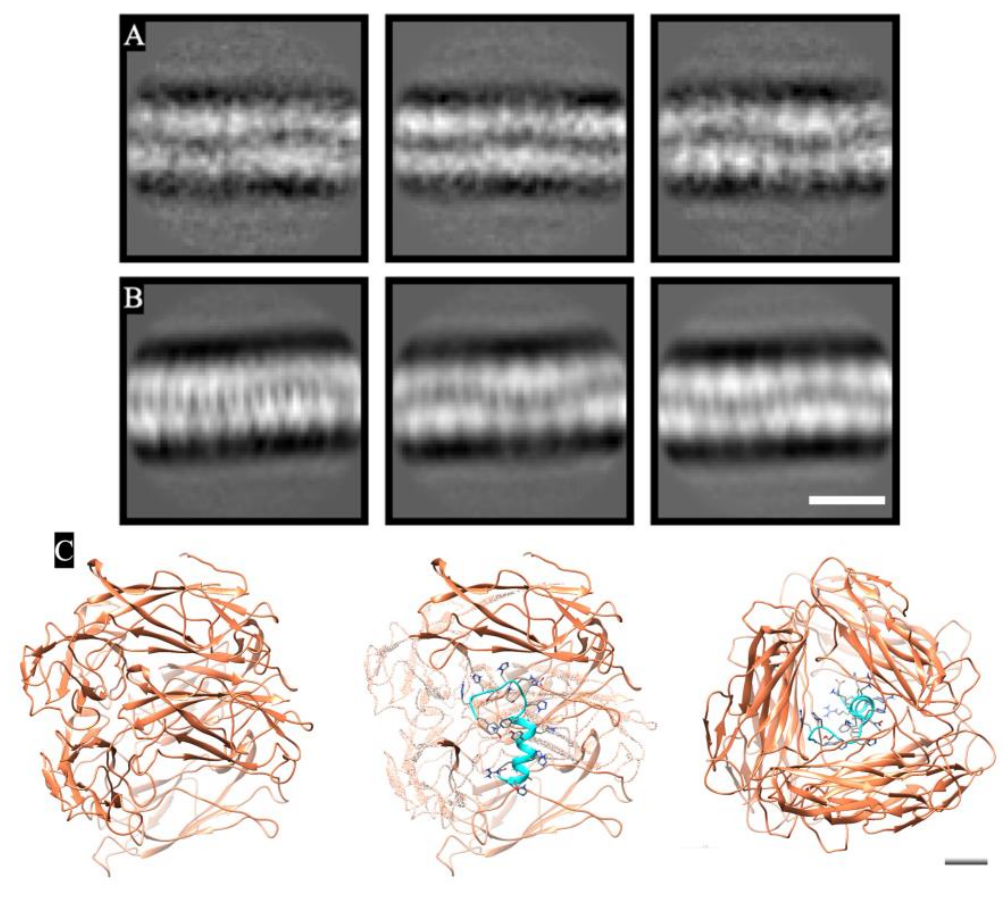

Figure 2. The central channel of CFA/I pili has density only in the presence of histatin-5, indicating an internal binding site for the salivary peptide. A, B). 2D reference-free class averages show density, presumed to be histatin-5, located in the hollow center channel of the CFA/I filament. Using Relion 3.1 software, individual filaments were manually picked from TF20 cryo-EM images of CFA/I pili in the absence and presence of histatin-5. Filament segments $215 \AA$ in length were extracted with $97 \%$ overlap and used to generate referencefree $2 \mathrm{D}$ class averages. In the absence of histatin-5, the center of the filament remains hollow. In the presence of histatin-5, conspicuous density that we attribute to the peptide appears in the previously hollow center. Scalebar, $5 \mathrm{~nm} . \mathrm{C}$ ). Protein-protein docking indicates a histatin-5 binding site in the center of the hollow CFA/I pilus. ClusPro, a protein docking program was used to optimize electrostatic and van der Waals potential interactions. Shown is the top-ranked proposed binding sites between the ligand histatin-5 and a six subunit CFA/I filament receptor site. The top ranked poses between histatin-5 and CFA/I pili appear within the filament's interior, with the most abundant interactions occurring between the first and third subunit. This histatin-5/pilus interaction increases the vertical, layer-to-layer interactions between pilin subunits.

\section{References}

1. Qadri, F., Svennerholm, A.-M., Faruque, A. S. G. \& Sack, R. B. Enterotoxigenic Escherichia coli in developing countries: epidemiology, microbiology, clinical features, treatment, and prevention. Clin. Microbiol. Rev. 18, 465-483 (2005).

2. Shah, N., DuPont, H. L. \& Ramsey, D. J. Global etiology of travelers' diarrhea: systematic review from 1973 to the present. Am. J. Trop. Med. Hyg. 80, 609-614 (2009). 
3. Checkley, W. et al. Multi-country analysis of the effects of diarrhoea on childhood stunting. Int. J. Epidemiol. 37, 816-830 (2008).

4. Satterwhite, T. K., Evans, D. G., DuPont, H. L. \& Evans, D. J. Role of Escherichia coli colonisation factor antigen in acute diarrhoea. Lancet Lond. Engl. 2, 181-184 (1978).

5. Sakellaris, H., Munson, G. P. \& Scott, J. R. A conserved residue in the tip proteins of CS1 and CFA/I pili of enterotoxigenic Escherichia coli that is essential for adherence. Proc. Natl. Acad. Sci. U. S. A. 96, 1282812832 (1999).

6. Zheng, W., Andersson, M., Mortezaei, N., Bullitt, E. \& Egelman, E. Cryo-EM structure of the CFA/I pilus rod. IUCrJ 6, 815-821 (2019).

7. Baker, J. L., Dahlberg, T., Bullitt, E. \& Andersson, M. Impact of an alpha helix and a cysteine-cysteine disulfide bond on the resistance of bacterial adhesion pili to stress. bioRxiv 2021.01.18.427124 (2021).

8. Brown, J. W. et al. A Role for Salivary Peptides in the Innate Defense Against Enterotoxigenic Escherichia coli. J. Infect. Dis. 217, 1435-1441 (2018).

9. Zivanov, J. et al. New tools for automated high-resolution cryo-EM structure determination in RELION-3. eLife 7, e42166 (2018).

10. Desta, I. T., Porter, K. A., Xia, B., Kozakov, D. \& Vajda, S. Performance and Its Limits in Rigid Body Protein-Protein Docking. Structure 28, 1071-1081.e3 (2020).

11. Brewer, D., Hunter, H. \& Lajoie, G. NMR studies of the antimicrobial salivary peptides histatin 3 and histatin 5 in aqueous and nonaqueous solutions. Biochem. Cell Biol. Biochim. Biol. Cell. 76, 247-256 (1998). 